\title{
Photoproduction of axionlike particles in the NA64 experiment
}

\author{
R. R. Dusaev, ${ }^{1, *}$ D. V. Kirpichnikov $\oplus^{2, \dagger}$ and M. M. Kirsanov $\oplus^{2, *}$ \\ ${ }^{1}$ National Research Tomsk Polytechnic University, 634050 Tomsk, Russia \\ ${ }^{2}$ Institute for Nuclear Research of the Russian Academy of Sciences, 117312 Moscow, Russia
}

(Received 25 May 2020; accepted 10 September 2020; published 28 September 2020; corrected 4 May 2021)

Axionlike particles $a$ (ALPs) that couple to the Standard Model gauge fields could be observed in the high-energy photon scattering $\gamma N \rightarrow N a$ off nuclei followed by the $a \rightarrow \gamma \gamma$ decay. In the present paper we describe the calculation of the ALP production cross section and the properties of this production. The cross section formulas are implemented in the program for the simulation of events in the NA64 experiment, the active electron beam dump facility at the CERN Super Proton Synchrotron. We study the prospects of the NA64 experiment to search for ALP in the $10 \mathrm{MeV} \lesssim m_{a} \lesssim 100 \mathrm{MeV}$ mass range for the statistics corresponding to up to $5 \times 10^{12}$ electrons on target.

DOI: 10.1103/PhysRevD.102.055018

\section{INTRODUCTION}

Axionlike particles (ALP) interacting with gauge bosons of the Standard Model (SM) arise naturally in various wellmotivated SM extensions such as string theory [1-3] and supersymmetry [4,5]. Being a pseudo Nambu-Goldstone boson of spontaneously broken global Peccei-Quinn symmetry [6], ALP originally addressed the strong-charge conjugation times parity problem [6-8]. More recently, the interest in a new light and weakly coupled pseudoscalar particle has been stimulated due to its relevance to the wellmotivated dark matter models [9-11].

The aim of the present work is to study the ALP production in the electron fixed target experiment NA64 at the CERN Super Proton Synchrotron (SPS) through the Primakoff reaction $\gamma N \rightarrow N a$. The NA64 (Fig. 1) is an active beam dump facility with a significant potential to probe various scenarios beyond the Standard Model. The well-motivated dark sector of particle physics has been already constrained by NA64 using the missing energy signatures [12-16].

Probing pseudoscalar particles in the $\mathrm{MeV}-\mathrm{GeV}$ mass range by the beam dump facilities is becoming a hot topic for experimental study. For instance, such planned experiments as FASER [17], MATHUSLA [18], SHIP [19], CodexB [20], SeaQuest [21] and LDMX [22] will be able to probe

\footnotetext{
renat.dusaev@cern.ch

${ }^{\dagger}$ kirpich@ms2.inr.ac.ru

*mikhail.kirsanov@cern.ch
}

Published by the American Physical Society under the terms of the Creative Commons Attribution 4.0 International license. Further distribution of this work must maintain attribution to the author(s) and the published article's title, journal citation, and DOI. Funded by SCOAP ${ }^{3}$. long-lived ALPs $[23,24]$ due to large distances between the ALP production vertex and detector. In these experiments, the ALP propagates typically along a distance of 10-100 m before its decay. This implies that the above-mentioned experimental facilities are sensitive to relatively small couplings in the range $10^{-7} \mathrm{GeV}^{-1} \lesssim g_{\text {ary }} \lesssim 10^{-4} \mathrm{GeV}^{-1}$. On the other hand, the typical decay length in the NA64 experiment is several meters depending on the NA64 geometry configuration. Therefore, due to this shorter length in this experiment it is possible to search for decays of ALPs with $g_{a \gamma \gamma} \gtrsim 10^{-4} \mathrm{GeV}$ for sub-GeV $m_{a}$. In addition, it is possible to search for long-lived ALPs in the missing energy signatures.

The paper is organised as follows. In Sec. II, we discuss the properties of the ALP. In Sec. III, we review the ALP production cross section in the Primakoff reaction. In Sec. IV, we discuss the Monte Carlo (MC) simulation of the ALP production in the NA64 experiment. In Sec. V, we describe the ALP search strategy. In Sec. VI, we estimate the expected sensitivity of the NA64 facility to the ALP for the statistics up to $5 \times 10^{12}$ electrons on target. We conclude in Sec. VII.

\section{THE ALP PROPERTIES}

We consider the simplified setup [25] of ALP coupling predominantly to photons:

$$
\mathcal{L}_{\text {int }} \supset-\frac{1}{4} g_{a \gamma \gamma} a F_{\mu \nu} \tilde{F}^{\mu \nu}+\frac{1}{2}\left(\partial_{\mu} a\right)^{2}-\frac{1}{2} m_{a}^{2} a^{2},
$$

where $F_{\mu \nu}$ denotes the strength of the photon field, and the dual tensor is defined by $\tilde{F}_{\mu \nu}=\frac{1}{2} \epsilon_{\mu \nu \lambda \rho} F^{\lambda \rho}$. We assume throughout the paper that the effective coupling $g_{a \gamma \gamma}$ and the 


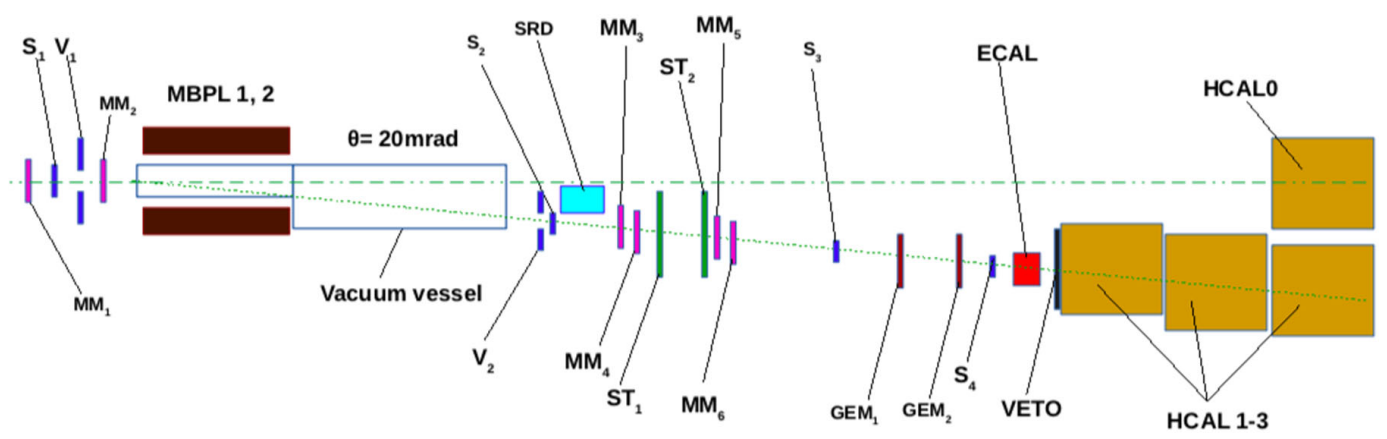

FIG. 1. NA64 configuration used for the search of ALP decays $a \rightarrow \gamma \gamma$.

ALP mass $m_{a}$ are independent. The pseudoscalar boson coupled to photons (1) has the following decay width:

$$
\Gamma_{a \rightarrow \gamma \gamma}=\frac{g_{a \gamma \gamma}^{2} m_{a}^{3}}{64 \pi} .
$$

The decay length of the ALP is given by

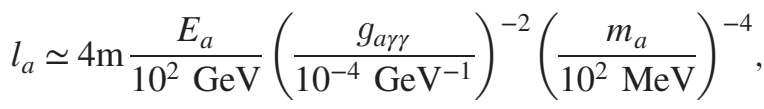

where $E_{a}$ is the ALP energy. The minimal decay length to which the NA64 facility is sensitive is of the order of the target thickness $(0.5 \mathrm{~m})$. Therefore, from Eq. (3) one can conclude that NA64 with a most used beam energy of $100 \mathrm{GeV}$ is sensitive to the values of ALP coupling to photons of the order of $g_{\text {ary }} \gtrsim 10^{-4} \mathrm{GeV}^{-1}$.

\section{CROSS SECTION}

We first calculate the cross section of axions produced in the Primakoff process $\gamma N \rightarrow a N$. The ALP production amplitude is given by

$$
\mathcal{M}=g_{a \gamma \gamma} e F\left(q^{2}\right) \epsilon_{\mu \nu \lambda \rho} \epsilon_{i}^{\mu}(p) p^{\lambda} q^{\rho}\left(\mathcal{P}_{i}+\mathcal{P}_{f}\right)^{\nu} \frac{1}{q^{2}},
$$

where $p, \mathcal{P}_{i}, \mathcal{P}_{f}$, and $k$ are the four-momenta of the incident photon, initial nucleus, final state nucleus, and the axion, respectively; note that $e$ is the electron charge. The internal photon momentum is defined by $q=\mathcal{P}_{i}-\mathcal{P}_{f}$. In Eq. (4) we suppose that the nucleus has spin zero, thus the corresponding nuclear-photon vertex is given by [26-29]

$$
i e F\left(q^{2}\right)\left(\mathcal{P}_{i}+\mathcal{P}_{f}\right) .
$$

The form-factor $F\left(q^{2}\right)$ depends upon the value of momentum transfer $q^{2}=-t$ and describes the elastic photon scattering [30] as

$$
F(t) \approx Z\left(\frac{a^{2} t}{1+a^{2} t}\right)\left(\frac{1}{1+t / d}\right)
$$

where $a=111 Z^{-1 / 3} / m_{e}$ and $d=0.164 \mathrm{GeV}^{2} A^{-2 / 3}$; here, $m_{e}$ is the mass of the electron and $A$ is the atomic weight. The inelastic form factor proportional to $\sqrt{Z}$ is small as compared to (5) for the high- $Z$ target material and thus yields a subdominant contribution to the ALP production that we neglect. The differential cross section of the elastic processes $\gamma N \rightarrow N a$ in the lab frame is given by

$$
d \sigma=\frac{1}{2^{5} \pi} \frac{1}{E_{\gamma}^{2} M_{N}} \overline{|\mathcal{M}|^{2}} d E_{a}
$$

where $E_{\gamma}$ is the incoming photon energy, and $M_{N}$ is the mass of the nucleus. The amplitude squared (see, e.g., Eq. (4) for details) averaged over the initial photon polarizations is given by

$$
\begin{aligned}
\overline{|\mathcal{M}|^{2}}= & \frac{1}{2} \sum_{\mathrm{pol}}|\mathcal{M}|^{2} \\
= & g_{a \gamma \gamma}^{2} e^{2} F^{2}(q) M_{N}^{2} \frac{1}{2 t^{2}} \\
& \times\left[\left(4 E_{a}^{2} t-\left(m_{a}^{2}+t\right)^{2}\right)-\frac{2 E_{a} t\left(m_{a}^{2}-t\right)}{M_{N}}-\frac{m_{a}^{2} t^{2}}{M_{N}^{2}}\right],
\end{aligned}
$$

here, we use the FeynCalc package [31] of Wolfram Mathematica [32] that carries out a summation in $\overline{|\mathcal{M}|^{2}}$ over dump indices. The resulting amplitude squared is given by

$$
\overline{|\mathcal{M}|^{2}} \simeq g_{a \gamma \gamma}^{2} e^{2} F^{2}(t) M_{N}^{2} \times \frac{1}{2 t^{2}}\left(4 E_{a}^{2} t-m_{a}^{4}\right)
$$

where we suppose that $m_{a} \gg t$ and neglect the third and fourth terms of Eq. (7) since the target nuclei are rather heavy, $M_{N} \gg m_{a}$ and $M_{N} \gg \sqrt{t}$. The angle $\theta_{a}$ between the incoming photon and the ALP can be derived from the momentum conservation law. The latter implies the following expression: 
$\cos \theta_{a}=\frac{1}{2\left|\mathbf{p}_{a}\right| E_{\gamma}} \cdot\left(2 E_{a}\left(E_{\gamma}+M_{N}\right)-2 E_{\gamma} M_{N}-m_{a}^{2}\right)$.

For the NA64 experiment, we are mainly interested in high energy photons produced by $100 \mathrm{GeV}$ electrons in the lead target. This corresponds to small momentum transfers and to small angles of ALP emission. In particular, we consider the limit when $m_{a} \ll E_{a}$ and $\theta_{a} \ll 1$, then Eq. (8) implies that the photon energy can be expressed as

$$
E_{\gamma} \approx E_{a}+\frac{E_{a}^{2} \theta_{a}^{2}}{2 M_{N}}+\frac{m_{a}^{4}}{8 M_{N} E_{a}^{2}} .
$$

In this approach, the ALP energy can be rewritten as follows:

$$
E_{a} \approx E_{\gamma}-\frac{E_{\gamma}^{2} \theta_{a}^{2}}{2 M_{N}}-\frac{m_{a}^{4}}{8 M_{N} E_{\gamma}^{2}}
$$

We note that one should not neglect the second term in Eq. (10), which is naively associated with a typical angle of ALP emission. In particular, from Eq. (10) it follows that the momentum transfer squared can be approximated as

$$
t=-q^{2}=2 M_{N}\left(E_{\gamma}-E_{a}\right) \approx E_{\gamma}^{2} \theta_{a}^{2}+\frac{1}{4} \frac{m_{a}^{4}}{E_{\gamma}^{2}} .
$$

It is worth mentioning, however, that the realistic typical angle of ALP production depends also on the properties of atomic form factors (see, e.g., Eq. (17) below for details). Finally, one can obtain the momentum transfer distribution from Eqs. (6) and (11)

$$
\frac{d \sigma}{d t}=\frac{1}{2^{3}} \cdot g_{a \gamma \gamma}^{2} \alpha F^{2}(t) \cdot \frac{1}{t^{2}}\left(t-t_{\min }\right),
$$

where $t_{\min }=m_{a}^{4} /\left(4 E_{\gamma}^{2}\right)$. The differential cross section $d \sigma / d t$ has a peak at



$$
t^{*}=2 t_{\min }+1 / a^{2},
$$

which is associated with typical momentum transfers. In the left panel of Fig. 2, we show $d \sigma / d t$ as a function of $t$ for various masses $m_{a}$ and typical energies of incoming photons $E_{\gamma}$. We note that the maximum allowed value of momentum transfer is given by

$$
q_{\max }=\sqrt{t_{\max }}=\sqrt{2 M_{N}\left(E_{\gamma}-m_{a}\right)} .
$$

For the typical threshold energy of interest $E_{\gamma}>50 \mathrm{GeV}$, we have $q_{\max } \gg \sqrt{t_{\min }}$. From the left panel of Fig. 2, it is seen that the cross section of ALP production is highly suppressed in the region of this value. This means that one can set $t_{\max }=\infty$ in the integration of Eq. (12) over $t$. Thus, the total cross section of the Primakoff process is

$$
\sigma_{\mathrm{tot}} \simeq \frac{1}{2^{3}} g_{a \gamma \gamma}^{2} \alpha \int_{t_{\min }}^{\infty} \frac{d t}{t^{2}}\left(t-t_{\min }\right) F^{2}(t)
$$

For the typical wide range of ALP masses, $20 \mathrm{MeV} \lesssim$ $m_{a} \lesssim 100 \mathrm{MeV}$ and typical energies of photons, $50 \mathrm{GeV} \lesssim E_{\gamma} \lesssim 100 \mathrm{GeV}$, the parameters of lead form factors $(Z=82$ and $A=207)$ satisfy $d \gg t_{\min }$ and $d \gg 1 / a^{2}$. Given that approach, one has the following expression for the total cross section in the leading logarithmic order:

$$
\sigma_{\mathrm{tot}}=\frac{16 \pi \alpha}{m_{a}^{3}} \cdot \Gamma_{a \rightarrow \gamma \gamma} \cdot \frac{Z^{2}}{2}\left(\ln \left[\frac{d}{1 / a^{2}+t_{\min }}\right]-2\right) .
$$

The total cross section depends rather weakly on $m_{a}$ and $E_{\gamma}$ (see Fig. 3). Additionally, in Appendix the exact expression for the total cross section in Eq. (14) is presented. One can see from Eq. (A1) that Eq. (15) is a reasonable approximation of Eq. (14) with accuracy better than $1 \%$ for the lead



FIG. 2. Left: differential cross section versus momentum transfer squared. Right: differential cross section versus angle of ALP emission. All cross sections are calculated for lead target and $g_{a \gamma \gamma}=1 \mathrm{GeV}^{-1}$. 




FIG. 3. Total cross section versus incident photon energy for lead target and $g_{a \gamma \gamma}=1 \mathrm{GeV}^{-1}$.

form factor and ultrarelativistic ALPs with sub-GeV masses.

From Eqs. (6), (7), (10), and (11) we obtain

$$
d \sigma \approx \frac{1}{m_{a}^{3}} 16 \pi \alpha F^{2}(t) \Gamma_{a \rightarrow \gamma \gamma} \frac{\theta_{a}^{3} d \theta_{a}}{\left(\theta_{a}^{2}+\delta_{a}^{2}\right)^{2}},
$$

where $\delta_{a} \approx m_{a}^{2} /\left(2 E_{\gamma}^{2}\right)$ is a parameter that characterizes a typical angle between the beam line and the ALP momentum. This result coincides with $[25,33,34]$. We note that $d \sigma / d \theta_{a}$ has a peak at

$$
\theta_{a}^{*} \approx \frac{1}{a E_{\gamma}} \sqrt{3\left(1+a^{2} t_{\min }\right)} .
$$

This is a typical angle of the ALP (see the right panel of Fig. 2). For $a^{2} t_{\min } \gg 1$, it is proportional to $\delta_{a}$, and for $a^{2} t_{\min } \ll 1$ it is scaled as $\propto 1 /\left(a E_{\gamma}\right)$.

\section{CALCULATION OF THE ALP YIELD IN NA64}

In this section, we discuss the implementation of the code for the MC simulation of the ALP production that uses the formulas derived above in the full simulation program based on GEANT4 [35] for the NA64 experiment [14].

The photons that can produce ALPs originate from the bremsstrahlung radiation of electrons and positrons of the electromagnetic shower from the primary $100 \mathrm{GeV}$ electron beam absorbed in the target-calorimeter ECAL. The Primakoff process of ALP production $\gamma N \rightarrow a N$ in this program can occur along with other SM processes for all photons of the electromagnetic shower, if the photon energy $E_{\gamma}$ is above some threshold that corresponds to the minimal detectable ALP energy.

Now we describe the calculation of the ALP signal in NA64 at each step of the photon propagation in the target. The number of ALPs produced at the $i$ th photon's step in the electromagnetic shower is

$$
N_{a}^{(i)}=\frac{\rho N_{A}}{A} \sigma_{\mathrm{tot}}\left(E_{\gamma}^{i}\right) \times l_{i},
$$

where $\rho$ is a density of the lead ECAL active target, $A$ is the atomic mass of the target, $N_{A}$ is Avogadro's number, $\sigma_{\text {tot }}\left(E_{\gamma}^{i}\right)$ is the total cross section of ALP elastic interaction with a nucleus (see, e.g., Eq. (15) for details), and $l_{i}$ is the step length of the photon in target.

In the simulation of signal samples, at each step of tracing of a photon with the energy above threshold the following actions (accept/reject scheme) are made:

(i) We randomly sample the variable $u$ distributed uniformly in the range [0,1]; if $u$ is smaller than the $a$ emission probability

$$
P_{\text {emission }}=\frac{\rho N_{A}}{A} \times \sigma_{\text {tot }}\left(E_{\gamma}^{i}\right) \times l_{i},
$$

then the emission of $a$ is accepted.

(ii) For each emitted $a$, we then generate the value of $E_{a} / E_{\gamma}$ and the angle of $a$ with respect to the initial photon according to the differential cross section (Fig. 2), then we calculate the four-momentum of the ALP. The value of $E_{a} / E_{\gamma}$ is very close to unity $E_{a} / E_{\gamma} \simeq 1$.

(iii) After production, the ALP decay is simulated according to Eq. (2).

In order to simulate samples with sufficient total statistics, we used the CERN batch system. This production process was automatized [36].

\section{THE ALP SEARCH STRATEGY}

We assumed the configuration of NA64 [16] initially designed for the search for invisible decays of dark photons $A^{\prime}$, which is suitable as well for the ALP search (see Fig. 1). In this configuration the electromagnetic calorimeter (ECAL), which serves as an active target, is followed by three modules of the hadron calorimeter (HCAL), denoted as HCAL1, HCAL2, HCAL3. The $100 \mathrm{GeV}$ beam of electrons is cleaned from other particles by the two dipole particle bending magnets (MBPL) and the synchrotron radiation detector (SRD) to the level of $10^{-6}$. The momentum of the incident electrons is measured with accuracy $\simeq 1 \%$.

Two distinct signatures of the ALP in the NA64 experiment are possible. In the first signature (visible signature in Fig. 4), the ALP decays in the second and third modules of HCAL, the first module (HCAL1) serving as a veto. So we required the energy deposition compatible with noise in HCAL1 (below $1 \mathrm{GeV}$ ) and at least $15 \mathrm{GeV}$ in HCAL2 and HCAL3. In addition, the energy deposition distributions in HCAL2 and HCAL3 should be compatible with two nearly collinear photons from the ALP decay $a \rightarrow \gamma \gamma$, very close to the electron beam axis because of very small angles of the ALP emission. This means that 



FIG. 4. NA64 design for the search for ALP decays $a \rightarrow \gamma \gamma$. Left panel corresponds to the visible signature in NA64, where ALPs decay in the central cells of the hadron calorimeter 2 (HCAL) and HCAL3 with HCAL1 being a veto. Right panel is the invisible signature in NA64, in which we search for decays $a \rightarrow \gamma \gamma$ outside all NA64 subdetectors.

almost all energy (more than 95\%) should be deposited in the central cell of the HCAL modules. This is important for the background suppression because hadronic showers are usually much wider and deposit significant energy in peripheric cells of HCAL. We also require that the energy is conserved, taking into account the energy resolution of calorimeters

$$
\left|E_{\mathrm{ECAL}}+E_{\mathrm{HCAL}}-E_{0}\right| \lesssim 10 \mathrm{GeV}
$$

where $E_{\mathrm{ECAL}}$ and $E_{\mathrm{HCAL}}$ are the energy depositions in electromagnetic and hadronic calorimeters, respectively, and $E_{0} \simeq 100 \mathrm{GeV}$ is the energy of primary electrons. For this signature, we do not apply any additional cut on $E_{\mathrm{ECAL}}$, accepting all events that passed the normal hardware trigger cut used in NA64 in all physical runs,

$$
E_{\mathrm{ECAL}} \lesssim 80 \mathrm{GeV} .
$$

The corresponding signal box is shown in Fig. 5.

In the second signature (invisible signature), the ALP decays beyond all subdetectors of NA64. This is the missing energy signature of the ALP, the same that is described in Refs. [13,16]. The selection criteria can be found in the corresponding references. The most important cuts are the upper ECAL energy cut and the requirement of no energy deposition in all three HCAL modules, HCAL1-HCAL3,

$$
E_{\mathrm{ECAL}} \lesssim 50 \mathrm{GeV}, \quad E_{\mathrm{HCAL}} \lesssim 1 \mathrm{GeV} .
$$

In the missing energy signature, the cut on the energy in the ECAL is rather strict. This means that only shower photons with the energy above $50 \mathrm{GeV}$ can produce detectable ALPs. This signature is shown in the right panel of Fig. 4. The corresponding signal box is shown in Fig. 5.

Note that the background conditions in the visible signature are much better, and for this reason it was possible to relax the cut on $E_{\mathrm{ECAL}}$ to $80 \mathrm{GeV}$ as compared to the invisible one. Correspondingly, in this signature photons with the energy as low as $\simeq 20 \mathrm{GeV}$ can produce detectable ALPs. The flux of such photons is significantly higher than thus above $50 \mathrm{GeV}$, detectable in the invisible signature. For this reason, the contribution of the visible signature to the total sensitivity of NA64 to ALPs is significant. In the signal samples, we simulated the ALP with the energy $E_{\mathrm{ALP}}>18 \mathrm{GeV}$ decaying beyond the HCAL1 module, which includes also $a \rightarrow \gamma \gamma$ decays far from the NA64 detectors. The cuts corresponding to the two signatures were applied during the processing of these samples by the reconstruction program.



FIG. 5. Signal boxes for ALP searches at NA64. For the visible signature, it is constructed according to the energy conservation $E_{0} \simeq E_{\mathrm{ECAL}}+E_{\mathrm{HCAL}}$ law. In the invisible signature, a small energy in HCAL and a significant missing energy are required $E_{\text {miss }}=E_{0}-\left(E_{\mathrm{ECAL}}+E_{\mathrm{HCAL}}\right)$. Here, $E_{\mathrm{HCAL}}=E_{\mathrm{HCAL} 1}+$ $E_{\mathrm{HCAL} 2}+E_{\mathrm{HCAL} 3}$ is the total energy deposition in all HCAL modules. For the illustrative purpose, we increase the signal box of invisible signature by factor of 5 along the $E_{\mathrm{HCAL}}$ axis. 
The background in the visible signature is caused mainly by the punchthrough leading $K_{0}$ and neutrons produced in electronuclear interactions in ECAL [37]. The background in both signatures is shown to be smaller than 0.2 events $[16,37]$, so that it can be neglected in the sensitivity estimates since the difference from the background free case is small. After the upgrade of the NA64 detector in 2020-2021 it will be further suppressed.

In the real experiment, the simultaneous statistical analysis of both signatures is to be performed. However, for the sensitivity estimation in the conditions of small background, we can simply sum up the numbers of expected signal events for visible and invisible signatures.

\section{THE EXPECTED SENSITIVITY OF NA64}

Now we estimate the sensitivity of NA64 to ALPs. The number of detectable ALPs can be written as

$$
N_{a}=\frac{N_{\mathrm{EOT}}}{N_{\mathrm{MC}}} \sum_{i} N_{a}^{(i)} \exp \left(-L_{D}^{(i)} / l_{a}^{(i)}\right) \mathcal{B}(a \rightarrow \gamma \gamma),
$$

where $N_{\text {EOT }}$ is the number of electrons on target in the experiment, $N_{\mathrm{MC}}$ is the number of simulated events, $L_{D}^{(i)}$ is the distance from the production point to the minimal allowed decay point coordinate, and $Z_{\min }, l_{a}^{(i)}$ is the ALP decay length taking into account its Lorentz factor [see, e.g., Eq. (3)]. Note that $Z_{\min }$ can be the end of HCAL1 or the end of HCAL3 depending on the signature under study. The typical lengths here are the lengths of the calorimeters $L_{\mathrm{ECAL}}=45 \mathrm{~cm}$ and $L_{\mathrm{HCAL} \text { module }}=1.3 \mathrm{~m}$. The typical energy of the ALP in the Primakoff process is $E_{a} \approx E_{\gamma}$, therefore, the ALP spectra are associated with the spectra of shower photons in the dump.

In Fig. 6 we show the number of $a \rightarrow \gamma \gamma$ decays as a function of ALP coupling with photons. Assuming background free case and zero signal events observed at NA64,

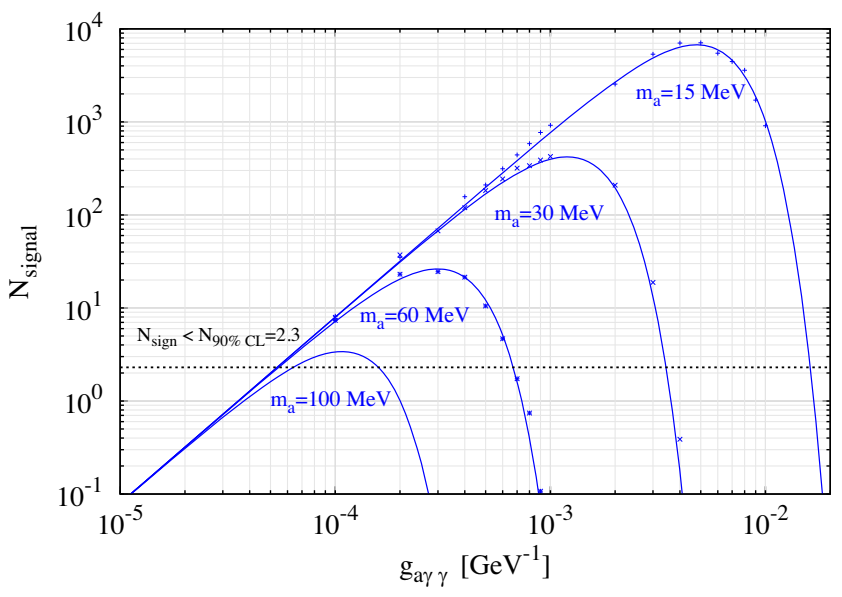

FIG. 6. Number of signal events as a function of $g_{\text {ary }}$ for $N_{\text {EOT }}=5 \times 10^{12}$.

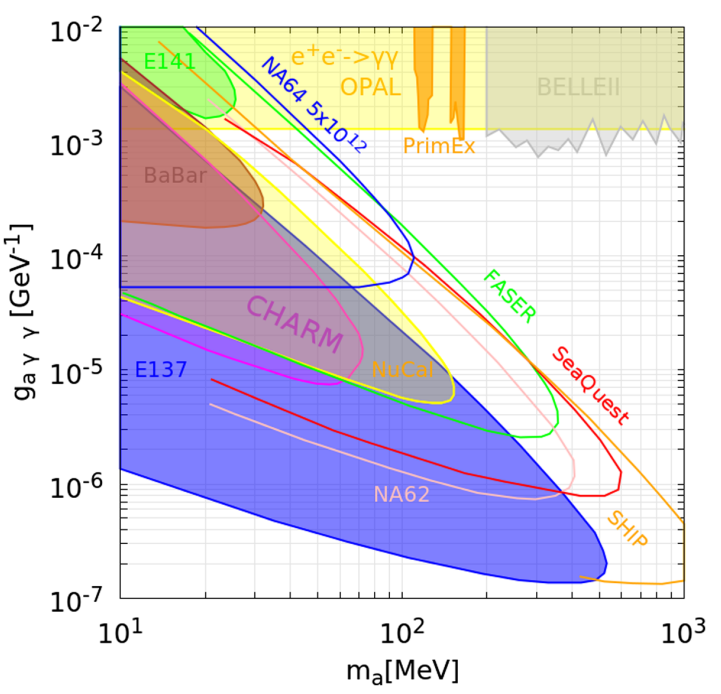

FIG. 7. The expected $90 \%$ C.L. sensitivity region of NA64 to the ALP production in the Primakoff process $\gamma N \rightarrow a N$ followed by the decay into photon pairs $a \rightarrow \gamma \gamma$ (solid blue line). The limits for E137 [33], CHARM [38], NuCal [39], BABAR [40], E141 [41], LEP [42] $\left(e^{+} e^{-} \rightarrow \gamma \gamma\right)$ and PrimEx [43] are taken from Refs. [44-46]. Recent bounds on ALPs from BELLEII [47] are shown by grey region. We also show the expected limits for FASER [48], NA62 [45], SeaQuest [21], and SHIP [25].

we require $90 \%$ C.L. upper limit on the number of ALP decays to be $N_{90 \%}=2.3$ according to the Poisson statistics. For each ALP mass $m_{a}$, the range of couplings constrained $g_{a \gamma \gamma}^{\text {low }}\left(m_{a}\right)<g_{a \gamma \gamma}<g_{a \gamma \gamma}^{\text {up }}\left(m_{a}\right)$ is defined by inequality $N_{a}>$ $N_{90 \%}$ (see Fig. 6). The values above $g_{a \gamma \gamma}^{\text {up }}$ correspond to short-lived ALPs decaying prematurely, before reaching the veto. The values below $g_{a \gamma \gamma}^{\text {low }}$ correspond to too small signal yield. The resulting plot in Fig. 6 includes both visible and invisible signatures.

In Fig. 7 we show the $90 \%$ C.L. sensitivity region of the NA64 experiment in the ALP parameters space for the background free case and the total number of $100 \mathrm{GeV}$ electrons on target $N_{\mathrm{EOT}}=5 \times 10^{12}$ in the mass range $10 \mathrm{MeV} \lesssim m_{a} \lesssim 100 \mathrm{MeV}$. The additional inefficiency of the detector due to instrumental effects not included in the MC-simulation is assumed to be negligible. It was below $20 \%$ in the published result [37] and will be significantly decreased after the detector upgrade in 2020-2021.

These results demonstrate that the NA64 experiment is capable to probe the ALP coupling with photons in the

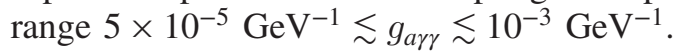

\section{CONCLUSION}

In the present manuscript, we have studied the prospects of the fixed target experiment NA64 that uses the electron beam at the CERN SPS to search for axionlike particles. In particular, we have studied the properties of the ALP production in the Primakoff reaction $\gamma N \rightarrow N a$ and its decay. We have implemented the ALP production cross 
sections and the process of its decay in the NA64 simulation program based on the GEANT4 toolkit. We have calculated the expected sensitivity to the ALP of the NA64 experiment and have shown that it potentially allows to examine the unexplored region in the parameter

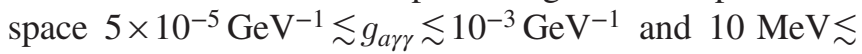
$m_{a} \lesssim 100 \mathrm{MeV}$, if the statistics corresponding to $N_{\mathrm{EOT}}=$ $5 \times 10^{12}$ electrons on target are accumulated.

\section{ACKNOWLEDGMENTS}

We would like to thank our colleagues from the NA64 Collaboration, in particular, S. N. Gninenko, N. V. Krasnikov, P. Crivelli, D. S. Gorbunov, and V.E. Lyubovitskij for useful discussions. This work was supported by the Ministry of Science and Higher Education (MSHE) in the frame of the Agreement No. 075-15-2020718, ID No. RFMEFI61320X0098.

\section{APPENDIX: EXACT FORMULA FOR THE TOTAL CROSS SECTION}

The full analytical expression for the integral (14) is

$$
\begin{aligned}
\sigma_{\mathrm{tot}}= & \frac{16 \pi^{2}}{m_{a}^{3}} \Gamma_{a \rightarrow \gamma \gamma} \frac{Z^{2}}{2} \frac{d^{2}}{\left(d-1 / a^{2}\right)^{3}} \\
& \times\left[\left(d+2 t_{\min }+1 / a^{2}\right) \log \left(\frac{d+t_{\min }}{1 / a^{2}+t_{\min }}\right)-2 d+2 / a^{2}\right] .
\end{aligned}
$$

For the parameter space of interest, $d \gg 1 / a^{2}$ and $d \gg t_{\min }$, the difference between the analytical (A1) and approximate expressions (15) is below $1 \%$.
[1] P. Svrcek and E. Witten, J. High Energy Phys. 06 (2006) 051.

[2] L. Visinelli and S. Vagnozzi, Phys. Rev. D 99, 063517 (2019).

[3] A. Arvanitaki, S. Dimopoulos, S. Dubovsky, N. Kaloper, and J. March-Russell, Phys. Rev. D 81, 123530 (2010).

[4] A. E. Nelson and N. Seiberg, Nucl. Phys. B416, 46 (1994).

[5] J. Bagger, E. Poppitz, and L. Randall, Nucl. Phys. B426, 3 (1994).

[6] R. D. Peccei and H. R. Quinn, Phys. Rev. Lett. 38, 1440 (1977).

[7] S. Weinberg, Phys. Rev. Lett. 40, 223 (1978).

[8] F. Wilczek, Phys. Rev. Lett. 40, 279 (1978).

[9] C. Boehm and P. Fayet, Nucl. Phys. B683, 219 (2004).

[10] M. J. Dolan, F. Kahlhoefer, C. McCabe, and K. SchmidtHoberg, J. High Energy Phys. 03 (2015) 171; 07 (2015) 103(E).

[11] Y. Hochberg, E. Kuflik, R. Mcgehee, H. Murayama, and K. Schutz, Phys. Rev. D 98, 115031 (2018).

[12] S. N. Gninenko, N. V. Krasnikov, M. M. Kirsanov, and D. V. Kirpichnikov, Phys. Rev. D 94, 095025 (2016).

[13] D. Banerjee et al. (NA64 Collaboration), Phys. Rev. Lett. 118, 011802 (2017).

[14] S. N. Gninenko, D. V. Kirpichnikov, M. M. Kirsanov, and N. V. Krasnikov, Phys. Lett. B 782, 406 (2018).

[15] S. N. Gninenko, D. V. Kirpichnikov, and N. V. Krasnikov, Phys. Rev. D 100, 035003 (2019).

[16] D. Banerjee et al. (NA64 Collaboration), Phys. Rev. Lett. 123, 121801 (2019).

[17] A. Ariga et al. (FASER Collaboration), Phys. Rev. D 99, 095011 (2019).

[18] J. P. Chou, D. Curtin, and H. J. Lubatti, Phys. Lett. B 767, 29 (2017).

[19] M. Anelli et al. (SHiP Collaboration), arXiv:1504.04956.
[20] V. V. Gligorov, S. Knapen, M. Papucci, and D. J. Robinson, Phys. Rev. D 97, 015023 (2018).

[21] A. Berlin, S. Gori, P. Schuster, and N. Toro, Phys. Rev. D 98, 035011 (2018).

[22] T. Akesson et al. (LDMX Collaboration), arXiv: 1808.05219 .

[23] J. Beacham et al., J. Phys. G 47, 010501 (2020).

[24] R. Alemany et al., arXiv:1902.00260.

[25] B. Dobrich, J. Jaeckel, F. Kahlhoefer, A. Ringwald, and K. Schmidt-Hoberg, J. High Energy Phys. 02 (2016) 018.

[26] Y. S. Liu and G. A. Miller, Phys. Rev. D 96, 016004 (2017).

[27] Y. S. Liu, D. McKeen, and G. A. Miller, Phys. Rev. D 95, 036010 (2017).

[28] T. Beranek and M. Vanderhaeghen, Phys. Rev. D 89, 055006 (2014).

[29] T. Beranek, H. Merkel, and M. Vanderhaeghen, Phys. Rev. D 88, 015032 (2013).

[30] J. D. Bjorken, R. Essig, P. Schuster, and N. Toro, Phys. Rev. D 80, 075018 (2009).

[31] V. Shtabovenko, R. Mertig, and F. Orellana, Comput. Phys. Commun. 207, 432 (2016).

[32] Wolfram Research, Inc., Mathematica, Version 12.1, Champaign, IL (2020), https://www.wolfram.com/ mathematica.

[33] J. D. Bjorken, S. Ecklund, W. R. Nelson, A. Abashian, C. Church, B. Lu, L. W. Mo, T. A. Nunamaker, and P. Rassmann, Phys. Rev. D 38, 3375 (1988).

[34] Y. S. Tsai, Phys. Rev. D 34, 1326 (1986).

[35] S. Agostinelli et al. (GEANT4 Collaboration), Nucl. Instrum. Methods Phys. Res., Sect. A 506, 250 (2003).

[36] https://gitlab.cern.ch/P348/na64-tools/tree/alps/geant4/ simulation/examples/example100500.

[37] D. Banerjee et al., Phys. Rev. Lett. 125, 081801 (2020). 
[38] F. Bergsma et al. (CHARM Collaboration), Phys. Lett. 157B, 458 (1985).

[39] J. Blümlein et al., Z. Phys. C 51, 341 (1991).

[40] B. Aubert et al. (BABAR Collaboration), arXiv:0808.0017.

[41] M. W. Krasny et al. (E141 Collaboration), University of Rochester Report No. UR-1029, 1987.

[42] G. Abbiendi et al. (OPAL Collaboration), Eur. Phys. J. C 26, 331 (2003).

[43] D. Aloni, C. Fanelli, Y. Soreq, and M. Williams, Phys. Rev. Lett. 123, 071801 (2019).

[44] M. J. Dolan, T. Ferber, C. Hearty, F. Kahlhoefer, and K. Schmidt-Hoberg, J. High Energy Phys. 12 (2017) 094.
[45] B. Dobrich, J. Jaeckel, and T. Spadaro, J. High Energy Phys. 05 (2019) 213.

[46] S. Knapen, T. Lin, H. K. Lou, and T. Melia, Phys. Rev. Lett. 118, 171801 (2017).

[47] Belle-II Collaboration, arXiv:2007.13071.

[48] J. L. Feng, I. Galon, F. Kling, and S. Trojanowski, Phys. Rev. D 98, 055021 (2018).

Correction: The affiliation for the first author contained an error and has been fixed. 